\title{
Expression patterns of Wnt signaling component, secreted frizzled-related protein 3 in astrocytoma and glioblastoma
}

\author{
NIVES PEĆINA-ŠLAUS ${ }^{1,2}$, ANJA KAFKA ${ }^{1,2}$, ANA MARIA VAROŠANEC ${ }^{1}$, \\ LEON MARKOVIĆ ${ }^{1}$, ŽELJKA KRSNIK $^{3}$, NIKO NJIRIĆ ${ }^{1}$ and GORAN MRAK ${ }^{4}$ \\ ${ }^{1}$ Laboratory of Neurooncology, Croatian Institute for Brain Research; ${ }^{2}$ Department of Biology, \\ School of Medicine, University of Zagreb; ${ }^{3}$ Department of Neuroscience, Croatian Institute for Brain Research, \\ School of Medicine, University of Zagreb; ${ }^{4}$ Department of Neurosurgery, University Hospital Center Zagreb, \\ School of Medicine, University of Zagreb, Zagreb HR-10000, Croatia
}

Received November 26, 2015; Accepted February 16, 2016

DOI: $10.3892 / \mathrm{mmr} .2016 .5061$

\begin{abstract}
Secreted frizzled-related protein 3 (SFRP3) is a member of the family of soluble proteins, which modulate the Wnt signaling cascade. Novel research has identified aberrant expression of SFRPs in different types of cancer. In the present study the expression intensities and localizations of the SFRP3 protein across different histopathological grades of astrocytic brain tumors were investigated by immunohistochemistry, digital scanning and image analysis. The results demonstrated that the differences between expression levels and malignancy grades were statistically significant. Tumors were classified into four malignancy grades according to the World Health Organization guidelines. Moderate $(\mathrm{P}=0.014)$ and strong $(\mathrm{P}=0.028)$ nuclear expression levels were significantly different in pilocytic (grade I) and diffuse (grade II) astrocytomas demonstrating higher expression values, as compared with anaplastic astrocytoma (grade III) and glioblastoma (grade IV). When the sample was divided into two groups, the moderate and high cytoplasmic expression levels were observed to be significantly higher in glioblastomas than in the group comprising astrocytoma II and III. Furthermore, the results indicated that high grade tumors were associated with lower values of moderate $(\mathrm{P}=0.002)$ and strong $(\mathrm{P}=0.018)$ nuclear expression in comparison to low grade tumors. Analysis of cytoplasmic staining demonstrated that strong cytoplasmic expression was significantly higher in the astrocytoma III and IV group than in the astrocytoma I and II group ( $\mathrm{P}=0.048)$. Furthermore, lower grade astrocytomas exhibited reduced membranous SFRP3 staining when compared with higher grade astrocytomas and this difference was statistically significant $(\mathrm{P}=0.036)$. The
\end{abstract}

Correspondence to: Dr Nives Pećina-Šlaus, Laboratory of Neurooncology, Croatian Institute for Brain Research, School of Medicine, University of Zagreb, Šalata 12, Zagreb HR-10000, Croatia E-mail: nina@mef.hr

Key words: astrocytic brain tumors, secreted frizzled-related protein 3 , Wnt signaling, glioblastoma present results demonstrated that SFRP3 protein expression levels were decreased in the nucleus in higher grade astrocytoma (indicating the expected behavior of an antagonist of Wnt signaling), whereas when the SFRP3 was located in the cytoplasm an increased expression level of SFRP3 was identified in the high grade astrocytomas when compared with those of a low grade. This may suggest that SFRP3 acts as an agonist of Wnt signaling and promotes invasive behavior.

\section{Introduction}

Astrocytomas are the most common type of primary central nervous system neoplasm worldwide and have been classified into four malignancy grades by the World Health Organisation (WHO) (1). Recently it has become evident that the growth of human astrocytic tumors is driven by complex signaling networks $(2,3)$. Malfunctions in Wnt signaling are responsible for the development of numerous types of cancer, and the present study proposes that aberrant Wnt signaling is important in the development and invasion of astrocytic brain tumors. Numerous novel findings, reported in the last two years, demonstrate that Wnt signaling is as important in glioma formation and invasiveness as other basic cellular pathways (2-4). Our previous studies recognized the involvement of Wnt signaling in astrocytomas $(5,6)$ and in particular showed that upregulation of transcription factors associated with the pathway, T-cell factor 1 (TCF1) and lymphoid enhancer-binding factor-1 (LEF1), is associated with higher malignancy grades (7).

The canonical Wnt signaling cascade is controlling events ranging from cell cycle regulation and embryonic cell fate determination to cell motility $(8,9)$. The signaling pathway is activated by the binding of different Wnt ligands to specific serpentine receptors, termed frizzleds (Fzs). As a consequence, the $\beta$-catenin levels rise, and $\beta$-catenin translocates to the nucleus where it binds to transcription factors $\operatorname{LEF} / \mathrm{TCF}(9,10)$. As a result of this binding, certain target genes are activated, including c-Myc, N-myc, c-Jun and cyclin D1, which reveals why constitutive activation of the Wnt pathway is responsible for tumorigenesis. A critical step in the inactivation of Wnt signaling involves the control 
of $\beta$-catenin degradation. The signaling pathway is inactive when low $\beta$-catenin expression levels are maintained (11).

Wnt signaling is regulated at various levels by a large number of molecular effectors. The modulating molecules function as either antagonists or agonists of the signaling, and fine-tuning of their association is particularly important for cell homeostasis and normal tissue functioning. Activation of Wnt signaling is controlled by different antagonists, among which are members of the secreted frizzled-related protein (SFRP) family. SFRPs are a family of soluble proteins known for their ability to inhibit the signaling pathway by binding to Wnt ligands and/or Fz receptors. In humans, the SFRP family numbers five of its members, of which SFRP3 is the orthologue of the founding member, frizzled-related protein B $(F r z B)(12-14)$. SFRPs were the first Wnt antagonists identified and they are structurally associated with Fz proteins. On the N-terminal these proteins (length, 300 amino acids) possess an Fz-like cysteine-rich domain (CRD), which displays similar sequence homology to the CRD domain on the extracellular aspect of the Fz receptors. However, unlike the Fz receptors, the SFRPs do not possess transmembrane or cytosolic domains. In front of the CRD domain there is a sequence for a signal peptide. In addition to the CRD region, SFRPs have a hydrophilic region on the C-terminal that appears to confer heparin-binding properties (14-16).

Previous studies demonstrated aberrant expression of SFRPs in different types of cancer $(17,18)$. Although SFRPs were initially considered to be tumor suppressors that exerted an inhibitory role in Wnt signaling (as they have been found to be downregulated in the majority of tumors investigated), novel findings report that SFRP also stimulates and, thus, activates the Wnt signaling pathway $(13,19)$. Furthermore, there are numerous reports regarding the overexpression of SFRPs in cancer, therefore this behavior can no longer be considered as inconsistent, but indicates that SFRPs exert a dual role in deleterious Wnt signaling $(18,19)$.

The molecular mechanisms and genetic landscapes of human astrocytic brain tumors require further elucidation. As malignant astrocytomas are characterized by diffuse infiltration of the surrounding non-neoplastic tissue, an explanation regarding the cell invasion processes is of particular importance. Therefore, the aim of the present study was to investigate and compare the expression intensity and localization of the SFRP3 protein within different histopathological grades of astrocytic brain tumor. The SFRP3 protein was evaluated, as there are various indications that it is associated with gliomas. A previous study has reported a downregulation of SFRP expression levels in various types of cancer, indicating a loss of function (18). Furthermore, an SFRP family member strongly promoted the growth of intracranial glioma xenografts in nude mice and promoted glioma cell growth in vitro (20). In addition, novel findings demonstrate SFRP3 as an important morphogen of mouse neurogenesis (21-23). However, to the best of our knowledge, the involvement of the SFRP3 protein has not been investigated in astrocytoma patients.

\section{Materials and methods}

Tumor specimens. Fifty-five astrocytic brain tumor samples were collected from the Hospital Centers, Sisters of Charity (Zagreb, Croatia) and the University Hospital Center Zagreb
(Zagreb, Croatia) between May 2007 and October 2015. The tumors were identified by magnetic resonance imaging in different cerebral regions. During surgery, the tumors were removed using a microneurosurgical technique. The patients had no family history of brain tumors, and all tumors were analyzed by pathologists and classified into four grades, according to WHO guidelines $(1,24)$. There were 10 pilocytic (grade I), 15 diffuse (grade II), and 11 anaplastic (grade III) astrocytomas and 19 glioblastomas (grade IV). There were 28 male patients and 27 female patients. The age of the patients ranged from 3 - to 73-years-old (mean age, 43.38 years; median age, 45.00 years). The mean age at diagnosis was 44.04 and 42.70 years for males and females, respectively. Ethical approval was obtained from the Ethical Committees Medical School University of Zagreb (Zagreb, Croatia), Hospital Center Sisters of Charity and University Hospital Center (380-59-10106-14-55/147) and the patients provided informed consent.

Immunohistochemistry. The samples were fixed in formalin, embedded in paraffin (both Kemika, Zagreb, Croatia), sliced into $4-\mu \mathrm{m}$ thick sections and fixed onto capillary-gap microscope slides (Dako, Glostrup, Denmark). The sections were immunostained using streptavidin-horseradish peroxidase/3,3'-diaminobenzidine (using EnVision ${ }^{\mathrm{TM}}$ REAL $^{\mathrm{TM}}$ detection systems; K5007; Dako). Briefly, sections were dewaxed by immersion in xylene (Kemika) twice for $5 \mathrm{~min}$. Subsequently, the sections were rehydrated in a descending ethanol dilution series (Kemika) and rinsed in $\mathrm{dH}_{2} \mathrm{O}$ for $5 \mathrm{~min}$. Sections were then microwaved twice for $10 \mathrm{~min}$ at $700 \mathrm{~W}$ in retrieval solution (S2369; Dako), cooled at room temperature for $15 \mathrm{~min}$, and microwaved once for $4 \mathrm{~min}$ at $350 \mathrm{~W}$ to unmask the epitopes. To block endogenous peroxidase activity, cells were fixed in methanol (Kemika) with $3 \% \mathrm{H}_{2} \mathrm{O}_{2}$. Non-specific binding was blocked by incubating samples with Protein Block, Serum-Free Ready-To-Use (Dako North America, Inc. Carpinteria, CA, USA) for $30 \mathrm{~min}$ at $4^{\circ} \mathrm{C}$. Next, the primary antibody, rabbit polyclonal anti-human FRP3 (1:50; sc-13941; Santa Cruz Biotechnology, Inc., Dallas, TX, USA) was applied for $30 \mathrm{~min}$ at room temperature. Slides were subsequently washed three times in phosphate-buffered saline (PBS; Amresco, Solon, $\mathrm{OH}, \mathrm{USA}$ )/goat serum, and the horseradish peroxidase-conjugated anti-rabbit/mouse secondary LINK antibodies from the Dako kit (K5007) were applied for $16 \mathrm{~min}$ at room temperature. Slides were washed a further three times in PBS/goat serum and were incubated with substrate chromogen solution (from the EnVision $^{\mathrm{TM}}$ REAL $^{\mathrm{TM}}$ kits) for $30 \mathrm{sec}$.

The sections were counterstained with Harris hematoxylin (Dako). Healthy brain and glioblastoma negative controls underwent the same staining procedure, but these samples were not incubated with primary antibodies. The frontal cortex of a healthy brain, and malignant melanoma and kidney tissue samples served as positive controls. Immunohistochemical staining was evaluated by assessing the staining intensity by three independent observers who were blinded to the experimental procedures. The staining intensity was scored as follows: No expression or very weak expression, $0 /+$; moderate expression, ++; and strong expression, +++. Two hundred cells in a hot spot, which is an area containing the most characteristics of malignant tissue and most active proliferative rate, of each sample were analyzed. The slides were scanned using a 

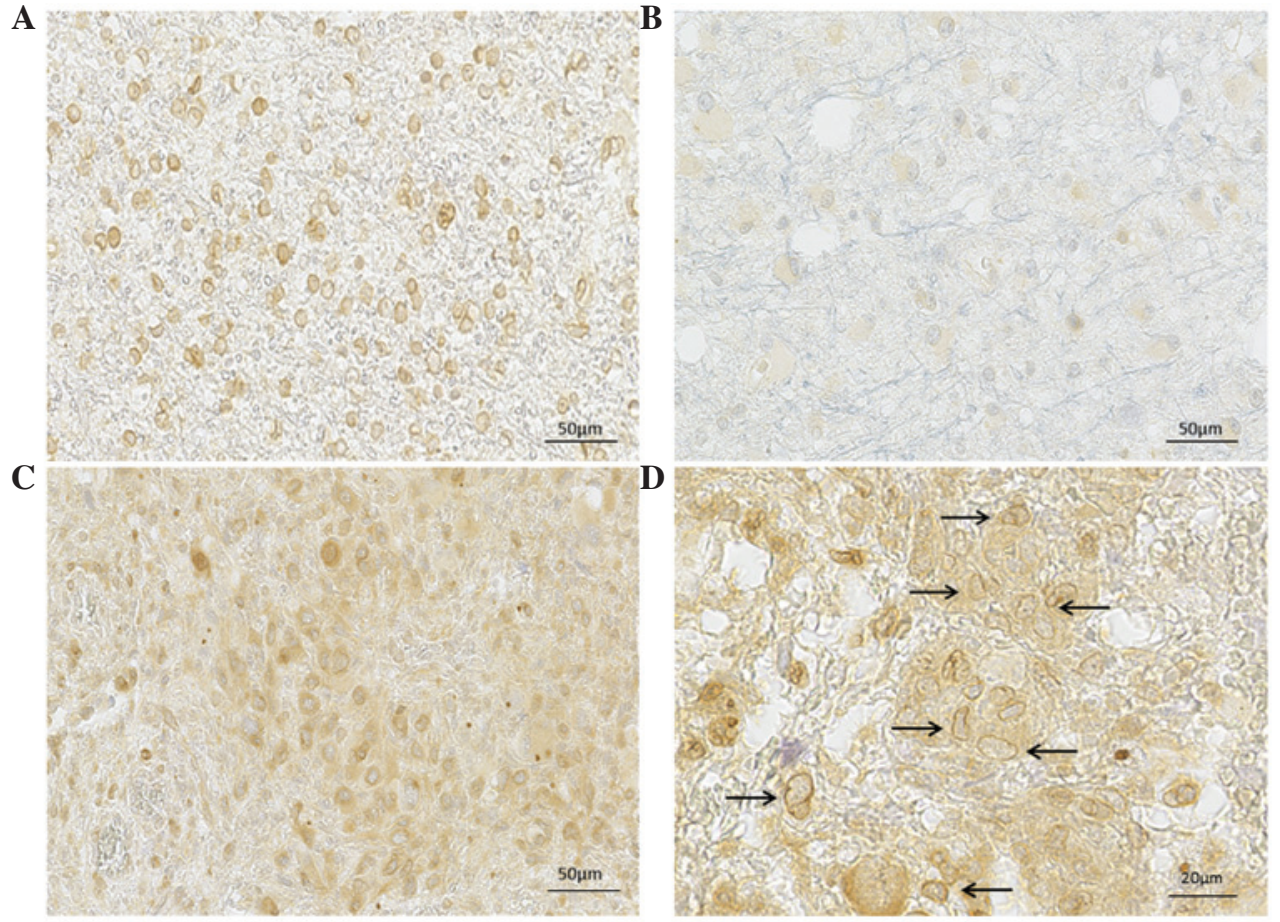

Figure 1. Characteristic immunohistochemical staining of different SFRP3 expression levels. (A) Diffuse astrocytoma showing strong nuclear staining, (B) anaplastic astrocytoma exhibiting moderate cytoplasmic expression, (C) glioblastoma demonstrating strong cytoplasmic staining, and (D) glioblastoma showing cytoplasmic and membranous staining (arrows). Scale bar: (A-C) $50 \mu \mathrm{m}$; (D) $20 \mu \mathrm{m}$.

digital scanner (NanoZoomer 2.0-RS; Hamamatsu Photonics, Hamamatsu, Japan), and ImageJ software (National Institutes of Health, Bethesda, Maryland, USA) was used to determine the cell number and the intensity of SFRP3 expression.

Statistical analysis. All individuals were analyzed for the following features: malignancy grade, gender, age, SFRP3 protein expression intensities and localizations. Differences in the values of SFRP3 expression levels (weak, moderate or strong) and the number of counted cells for each intensity were tested with analysis of variance (ANOVA) following Leven's analysis of homogeneity of variance (if significance of Leven's statistic was $<0.05$ the non-parametric Mann-Whitney test was employed). ANOVA was used to determine potential differences in the values of SFRP3 expression with regard to different malignancy grades, cellular localization, different age categories and gender. Student's t-test was used to analyze differences in membranous location. All statistical evaluations were performed using SPSS 14.0 (SPSS Inc., Chicago, IL, USA) and $\mathrm{P}<0.05$ was considered to indicate a statistically significant difference.

\section{Results}

Expression levels and localizations of SFRP3 protein. The SFRP3 protein, a molecule that is considered to have an antagonistic role in Wnt signaling, but has never been investigated in astrocytomas, was observed in the present study. On a sample of 55 astrocytic brain tumors, the expression and localization of SFRP3 was determined throughout different malignancy grades. SFRP3 was positively stained and localized in the nuclei and cytoplasm of tumor cells. Furthermore, different expression patterns were observed in nuclear and cytoplasmic localization, with varying staining intensities. Notably, specific cellular compartments demonstrated different intensities and, therefore, the separate staining intensities for cytoplasmic and nuclear compartments were assessed.

From the total sample, the mean number of cells with weak or no expression was 104; 25 and 61 cells (mean value) demonstrated moderate nuclear and cytoplasmic expression, respectively; and 18 and 41 cells (mean value) demonstrated strong nuclear and cytoplasmic expression, respectively (Fig. 1).

Expression levels and localizations in different malignancy grades. When the sample was divided according to malignancy grade, the differences in expression levels and localizations were significant. The levels of moderate and strong expression in the cell nucleus were associated with reduced numbers of cells in samples of higher malignancy grade tumors; whereas for cytoplasmic staining, the number of cells with strong SFRP3 expression was higher in astrocytoma grades III and IV when compared with samples from lower grade tumors.

When comparing the percentage of counted cells exhibiting low or no expression with a specific tumor grade, no statistically significant difference was identified between low nuclear or low cytoplasmic staining and the different grades $(\mathrm{F}=0.815 ; \mathrm{P}=0.491)$. However, when moderate and strong nuclear and cytoplasmic expression levels were investigated, the differences between expression levels and malignancy grade were statistically significant. Moderate nuclear expression levels were statistically different $(\mathrm{F}=3.874 ; \mathrm{P}=0.014)$, and grade I and II astrocytomas demonstrated the highest expression values: Grade I, 10.99; grade II, 14.14; grade III, 5.65; 
A

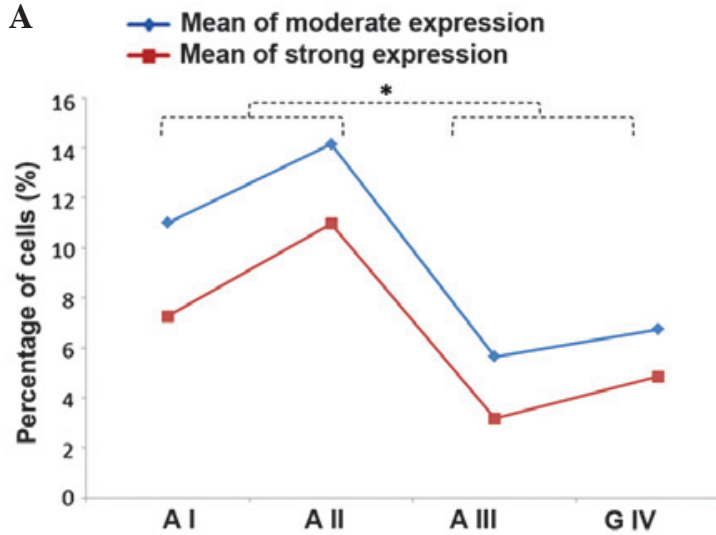

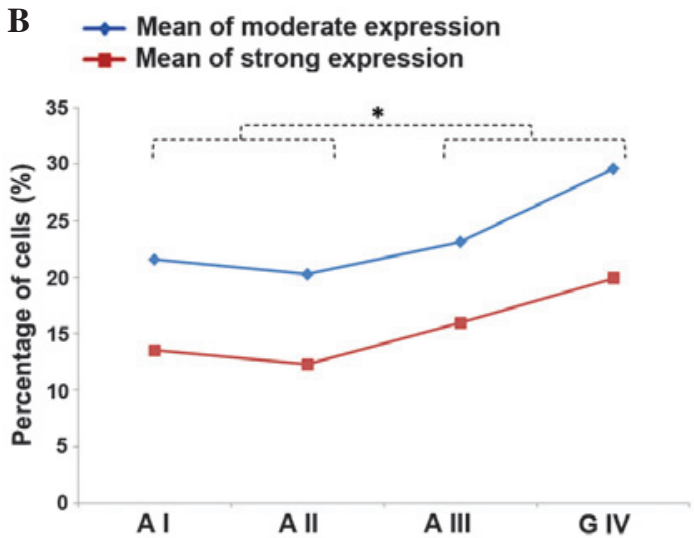

Figure 2. Graphs demonstrating moderate and strong levels of secreted frizzled-related protein 3 expression in different grades of astrocytic brain tumor (A) Nuclear and (B) cytoplasmic expression. (A) High grade tumors (A III and G IV) exhibited significantly lower mean values of both moderate and strong nuclear expression, as compared with the moderate and strong values of low grade tumors (A I and A II) ( $\left.{ }^{*} \mathrm{P}<0.05\right)$. (B) Mean values of moderate and strong cytoplasmic expression for high grade tumors were significantly higher than the mean values of low grade tumors $\left({ }^{*} \mathrm{P}<0.05\right)$. A I, pilocytic astrocytoma; A II, diffuse astrocytoma; A III, anaplastic astrocytoma; G IV, glioblastoma.

and grade IV, 6.73. Similarly, strong nuclear expression levels were different according to the malignancy grades $(\mathrm{F}=3.30$; $\mathrm{P}=0.028$ ) with grades I and II astrocytomas showing greater values: Grade I, 7.26; grade II, 10.96; grade III, 3.17; and grade IV, 4.85 . The association between nuclear moderate and strong staining in the different malignancy grades is presented in Fig. 2A.

Upon analysis of the cytoplasmic expression levels, moderate cytoplasmic expression was identified to be significantly different across the malignancy grades $(\mathrm{F}=2.319 ; \mathrm{P}=0.086)$, while strong cytoplasmic expression levels did not demonstrate a statistically significant difference $(\mathrm{F}=1.708 ; \mathrm{P}=0.177)$. The association between cytoplasmic moderate and strong staining in the different malignancy grades is presented in Fig. 2B.

SFRP3 expression levels and localizations in the two groups. Samples were then grouped according to clinical malignancy grades. First, the group of pooled astrocytoma II and III was examined in comparison with the group of grade IV with the highest malignancy grade. The nuclear expression levels between those two groups were not statistically different for the moderate and strong expression levels (Fig. 2A). However, a statistically significant difference was noted for the cytoplasmic expression levels. Values of moderate cytoplasmic expression were higher in grade IV (29.62) than in astrocytoma II and III (21.47) and this difference was significant $(\mathrm{F}=3.950 ; \mathrm{P}=0.053)$. The difference between strong cytoplasmic expression levels were also statistically significant between astrocytoma II and III, and IV $(\mathrm{F}=3.959 ; \mathrm{P}=0.05)$. Grade IV glioblastomas demonstrated higher values (19.92) when compared with astrocytoma grades II and III (13.84) (Fig. 2B).

The present study also aimed to investigate whether there is difference between low and high grade astrocytomas. Therefore, the sample was grouped into low grade (I and II) and high grade (III and IV) groups. Strong statistical differences for moderate and high nuclear expression levels were demonstrated (Fig. 2A). For moderate nuclear expression a significant difference was identified between the low and high grade astrocytomas $(\mathrm{F}=10.573 ; \mathrm{P}=0.002)$. The high grade tumors had lower values of moderate nuclear expression (6.33) when compared

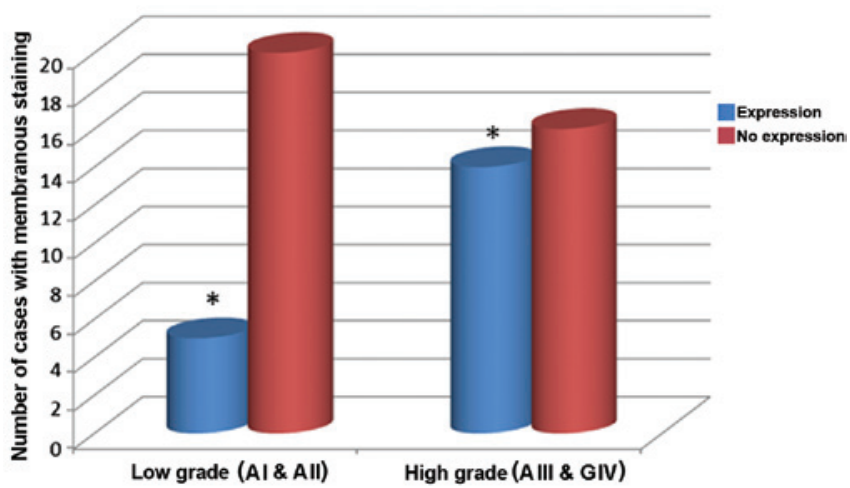

Figure 3. Low grade (A I and A II) group demonstrated significantly less membranous localization of secreted frizzled-related protein 3 when compared with the high grade (A III and G IV) group ( $(\mathrm{P}=0.036)$. A I, pilocytic astrocytoma; A II, diffuse astrocytoma; A III, anaplastic astrocytoma; G IV, glioblastoma.

with the low grade tumors (12.88). Strong nuclear levels were evaluated with the Mann-Whitney U test, which indicated that high tumor grades were associated with significantly lower levels of strong nuclear expression when compared with grade I and II astrocytomas $(\mathrm{P}=0.018)$. Analysis of the low and high grade astrocytoma groups using cytoplasmic staining showed that moderate cytoplasmic expression levels were significantly higher $(\mathrm{F}=3.381 ; \mathrm{P}=0.072)$ in the astrocytoma III and glioblastoma groups than in the astrocytoma I and II groups (Fig. 2B). Furthermore, strong cytoplasmic expression was significantly higher $(\mathrm{F}=4.116 ; \mathrm{P}=0.048)$ in the high grade tumor group (A III and G IV) as compared with the low grade group (A I and A II).

In addition, $34.5 \%$ of the samples demonstrated membranous localization of the signal. Positive membranous staining was observed in a relatively low number of cells per sample. However, the association of the membranous localization and the malignancy grades was investigated. It was demonstrated that lower grade astrocytomas exhibited reduced membranous staining when compared with higher grade astrocytomas, and that the difference between grades was significant ( $\mathrm{P}=0.036$; Fig. 3$)$. 
SFRP3 expression and epidemiological characteristics. The next step was to investigate the association between epidemiological characteristics of the astrocytoma patients and the SFRP3 expression levels. When the molecular findings were analyzed with the demographic variables it was not possible to demonstrate that the expressions levels and locations were associated with the analyzed age categories. However, statistically significant differences between moderate and strong nuclear expression levels between genders were established. Female patients with high grade astrocytomas (grades III and IV) showed higher moderate and strong nuclear expression levels than male patients, and this difference was significant (moderate: $\mathrm{F}=8.422$, $\mathrm{P}=0.007$; strong: $\mathrm{F}=9.262, \mathrm{P}=0.005$ ). Notably, no difference in cytoplasmic expression levels was identified between genders.

The results indicated that SFRP3 expression levels in the nucleus decreased in the higher astrocytoma grades, indicating the expected behavior as a tumor suppressor and an antagonist of Wnt signaling, whereas SFRP3 expression levels in the cytoplasm were increased in the high grade astrocytomas, compared with low grade astrocytomas. This may indicate that SFRP3 also acts as an agonist of Wnt signaling, promoting invasive behavior.

\section{Discussion}

SFRPs are a family of soluble proteins known for their ability to negatively modulate the Wnt signaling cascade. It has been found that this protein family is also involved in different types of cancer $(13,18)$. The present study demonstrated different expression patterns and staining intensities of the SFRP3 protein in a sample of astrocytic brain tumors of different pathohistological grades. The number of cells with low or no expression was not significantly different between the different tumor grades. However, when moderate and strong nuclear and cytoplasmic expression levels were investigated, the differences between expression levels and malignancy grade were statistically significant. Grade I and II astrocytomas demonstrated significantly higher expression values in moderate and strong nuclear expression. The analyses on cytoplasmic expression levels showed that moderate cytoplasmic expression was significantly differently distributed throughout the malignancy grades, whereas high cytoplasmic expression levels were not identified to be statistically different. However, when the sample was divided into two groups, one that included astrocytoma II and III and the other that included grade IV, statistical differences were observed for the moderate and high cytoplasmic expression levels. Furthermore, moderate and high cytoplasmic expression levels were significantly higher in grade IV when compared with astrocytoma II and III. Thus, demonstrating that SFRP3 may exert different effects; in the nucleus, SFRP3 functions as a tumor suppressor, while its cytoplasmic expression levels indicate oncogenic properties in higher grade astrocytomas. There are numerous papers that support SFRPs context-dependent dual role and are consistent with the present findings $(18,19)$. Hirata et al (17) investigated renal cell carcinoma and identified that the expression level of SFRP3 protein was decreased in primary renal cancer tissue samples when compared with normal kidney tissue samples; however, the level was restored in metastatic renal cancer tissues. In addition, the authors suggested that there may be a change in SFRP3 function, from that of a tumor suppressor to an oncogene, in renal cancer progression and metastasis. The present results are consistent with these findings, thus, it is hypothesized that the function of SFRP3 may alter during astrocytoma progression and the observed increase of cellular SFRP3 expression in glioblastoma in the current study may induce aggressive behavior and invasion. Additional studies regarding the dual role of SFRP in breast cancer demonstrate that SFRPs were highly overexpressed and associated with tumor progression $(18,25)$. In addition, endometrial cancer studies observed a dual role of SFRPs in Wnt signaling, with the majority supporting the notion that SFRPs may inhibit Wnt signaling. However, there are studies that have shown SFRP4 expression to be positively correlated with cancer malignancy (26). In ovarian cancer, SFRP4 expression tends to be downregulated, however, there are studies regarding the high expression of this protein in cancer tissue samples (27). Huang et al (28) demonstrated that there was an association between SFRP4, and risk of rectal cancer and early-stage colorectal cancer. In colorectal cancer patients, SFRP4 expression was significantly increased in the cancerous tissue samples when compared with the non-cancerous colorectal mucosa. The SFRP4 protein was upregulated in $45 \%$ of colorectal cancer tissue samples when compared with the matched non-cancerous tissue samples.

Astrocytomas are the most common type of brain tumor in humans, and glioblastoma are particularly proliferative and their invasive nature is correlated with particularly poor clinical outcomes $(29,30)$. In the present study SFRP3 expression was demonstrated to vary among different astrocytic malignancy grades. As pilocytic astrocytomas are considered to be clinically, biologically and histologically distinct from WHO grade II-IV gliomas, they may be regarded as a benign reference. However, according to the WHO classification and cellular characteristics pilocytic astrocytomas are described as low grade astrocytic tumors (grade I). Diffuse are also low grade (grade II), while anaplastic astrocytoma (grade III) and glioblastoma multiforme (grade IV) are classified as high grade astrocytomas. This classification was useful, as the SFRP3 protein showed marked differences between the low and high grade tumors. When the sample was split into low and high grade tumor groups, statistical differences for moderate and high nuclear expression levels were observed. High grade tumors exhibited lower values of moderate and strong nuclear expression when compared with low grade tumors. Cytoplasmic staining of high and low grade tumor groups showed that moderate cytoplasmic expression levels were significantly higher in the astrocytoma III and IV group when compared with the astrocytoma I and II group. Furthermore, strong cytoplasmic expression was identified to be significantly higher in the high grade tumors compared with the low grade tumors. Thus, in contrast to the normal antagonistic role, it was demonstrated that high cytoplasmic expression levels may act as a Wnt signaling activator and induce tumor invasion.

The function of SFRPs in tumor development also indicated that SFRPs do not always act as Wnt antagonists. Tissue culture experiments demonstrated that, at low concentrations, SFRP1 potentiates Wnt activity rather than inhibits it $(31,32)$. In combination with other observations, this finding has led to the suggestion that SFRP1 has low- and high-affinity binding sites for Wingless and Wnt ligands; binding to the high-affinity site promotes Wnt signaling, whereas binding to the low-affinity 
site inhibits it $(16,31)$. Gradient formation of expressed proteins must also be considered. Sun et al (23) identified that SFRP3 is expressed at a gradient (i.e. at different levels) along the septo-temporal axis of the dentate gyrus, which is established during postnatal development. Therefore, gradients of SFRP3 expression may contribute in various ways to cancer initiation and progression. It remains unclear whether SFRPs antagonize Wnt signaling by interacting with Wnt ligands via their N-terminal CRD or the C-terminal domain $(31,33)$. The conflicting data may result from differential affinities among SFRPs and their Wnt partners, or the use of different ligands. Thus, SFRPs may block Wnt signaling either by interacting with Wnt proteins, to prevent them from binding to Fz proteins, or by forming non-functional complexes with $\mathrm{Fz}(16,34)$.

The findings of the present study regarding membranous localization of SFRP3 and its significant prevalence in high grade tumors is consistent with the findings of high cytoplasmic expression levels in high grade tumors $(17-19,25,26,28)$. Therefore, it is proposed that events occurring at the cell surface are also important and may influence tumorigenesis and downstream cellular signaling. The observations from the current study of SFRP3 protein expression in the cellular membrane do not elucidate whether the SFRPs bind to Wnt or the receptor, however, do indicate localization that could make it a possibility. Statistical significance in moderate and strong nuclear expression levels between the genders was demonstrated. Female patients with high grade astrocytomas (grades III and IV) demonstrated higher nuclear expression levels than male patients.

Our previous study found $\beta$-catenin to be upregulated and transferred to the nucleus in astrocytic brain tumors (35). It was also demonstrated that the transcription factors of the Wnt signaling pathway were upregulated (7). Strong TCF1 and LEF1 expression levels were observed in 51.6 and $71.0 \%$ of glioblastomas, respectively. Astrocytoma grade I showed almost opposite expression levels, with weak or no expression of TCF1 and LEF1 in 63.2 and $68.2 \%$, respectively. Additionally, statistical analysis confirmed significant differences in protein expression levels and indicated that LEF1 may serve as a potential diagnostic marker distinguishing glioblastomas from astrocytomas (7). Wnt/ $\beta$-catenin signaling is essential for tumorigenesis, however its molecular mechanisms are not fully understood. Zhang et al (36) demonstrated upregulation of $\beta$-catenin in gliomas. Subsequently, it was discovered that Forkhead Box m1 (Foxm1)/ $\beta$-catenin interaction is required for glioma formation, and represents a mechanism for canonical Wnt signaling during tumorigenesis. The findings by Zhang et al (36) elucidate a mechanism for $\beta$-catenin nuclear translocation via binding to the transcription factor, Foxm1. Furthermore, this interaction is maintained in the nucleus, where the two proteins form a complex with TCF transcription factors on the promoters of $\mathrm{Wnt} / \beta$-catenin target genes. There are increasing studies investigating the role of the Wnt signaling pathway in human astrocytomas, however very few have investigated its role in progression. To the best of our knowledge, the role of SFRP3 has not yet been investigated in astrocytomas. Kahlert et al (37) demonstrated that Wnt signaling enhances motility of glioblastoma cells in vitro by activating molecules that promote the mesenchymal phenotype. In addition, the authors identified that the distribution of the nuclear $\beta$-catenin signal was predominantly within the invasive front of the tumor, which indicates that Wnt signaling is important in the regulation of malignant cell motility.

Studies regarding SFRP1 (4) have demonstrated a novel molecular miR-328-dependent mechanism, which via SFRP1 inhibition and Wnt activation contributes to the infiltrative glioma phenotype at early stages of glioma progression. The authors showed that a low SFRP1 expression level is a negative prognostic factor in gliomas. Roth et al (20) investigated SFRP1 and SFRP2 and demonstrated that the two proteins are produced by the majority of malignant glioma cell lines. It was found that SFRP2 promotes glioma cell growth in vivo and that these SFRPs were important modulators of the pathophysiology of malignant brain tumors.

The present results regarding SFRP3 expression levels and localizations provide novel insights into Wnt signaling changes in astrocytic brain tumors. Although it is easier to assign a single dimension to a certain protein, it appears that the majority of molecules possess numerous dimensions, and the current findings on SFRP3 support this hypothesis. Although SFRP3, also termed $F r z B$, has been associated with the inhibition of Wnt signaling, there are numerous congruous reports indicating that it activates Wnt signaling during progression and metastasis. Specific spatio-temporal dynamic expression of SFRP3, similar to a developmental gradient (22), is occurring in astrocytoma progression and glioblastoma development. Two distinct mechanisms participate in the loss of SFRP expression in cancer: Allelic loss and epigenetic silencing (14). It has been shown that the majority of SFRPs are epigenetically silenced via promoter hypermethylation in numerous types of cancer $(38,39)$. Although SFRP3 possesses no $\mathrm{CpG}$ islands in the promoter region, there have been no reports regarding the association between SFRP3 expression and epigenetic silencing; thus, it appears that its downregulation is achieved by another molecular mechanism.

Astrocytic brain tumors and, in particular, glioblastoma demonstrate great heterogeneity that has recently been explained by a sub-population of glioblastoma cancer stem cells, which are the predominant tumorigenic force (3). The Wnt signaling pathway is known to critically regulate self-renewal and differentiation of neural stem/progenitor cells $(3,40,41)$. Rheinbay et al (3) demonstrated that achaete-scute family bHLH transcription factor 1, a transcription factor essential for maintenance and in vivo tumorigenicity of glioblastoma cancer stem cells, activates Wnt signaling and that it is linked to the activation of LEF1. Rampazzo et al (2) described that Wnt activation promotes a marked differentiation of glioblastoma cancer stem cells towards a less aggressive phenotype.

In conclusion, the present results indicate that the decreasing SFRP3 expression level in the nucleus is positively correlated with increasing astrocytoma grade; whereas the increase in SFRP3 protein expression in the cytoplasm of higher grade astrocytomas demonstrates the dual nature of SFRP3. In certain cases, SFRP3 acts as an antagonist, while in other cases it serves as an agonist of the Wnt signaling pathway. The findings suggest that molecular changes in Wnt signaling are important in astrocytic tumor etiology. The novel molecular features may provide resources for future investigations regarding the pathogenesis mechanisms and tumor biology, and may facilitate with developing effective therapeutic strategies against this lethal type of cancer. SFRP3 may be adopted as a potential tool for combating Wnt driven tumorigenesis. 


\section{Acknowledgements}

The present study was supported by a grant from the Croatian Science Foundation (grant no. 6625).

\section{References}

1. Louis DN, Ohgaki H, Wiestler OD, Cavenee WK, Burger PC, Jouvet A, Scheithauer BW and Kleihues P: The 2007 WHO classification of tumours of the central nervous system. Acta Neuropathol 114: 97-109, 2007.

2. Rampazzo E,Persano L,Pistollato F, Moro E, Frasson C,Porazzi P, Della Puppa A, Bresolin S, Battilana G, Indraccolo S, et al: Wnt activation promotes neuronal differentiation of glioblastoma. Cell Death Dis 4: e500, 2013.

3. Rheinbay E, Suvè ML, Gillespie SM, Wakimoto $\mathrm{H}$, Patel AP, Shahid M, Oksuz O, Rabkin D, Martuza RL, Rivera MN, et al: An aberrant transcription factor network essential for Wnt signaling and stem cell maintenance in glioblastoma. Cell Rep 3: 1567-1579, 2013.

4. Delic S, Lottmann N, Stelzl A, Liesenberg F, Wolter M, Götze S, Zapatka M, Shiio Y, Sabel MC, Felsberg J, et al: MiR-328 promotes glioma cell invasion via SFRP1-dependent Wnt-signaling activation. Neuro Oncol 16: 179-190, 2014.

5. Nikuševa-Martić T, Beroš V, Pećina-Šlaus N, Pećina HI and Bulić-Jakuš F: Genetic changes of CDH1, APC, and CTNNB1 found in human brain tumors. Pathol Res Pract 203: 779-787, 2007.

6. Pećina-Šlaus N, Martić TN, Kokotović T, Kusec V, Tomas D and Hrasćan R: AXIN-1 protein expression and localization in glioblastoma. Coll Antropol 35 (Suppl 1): 101-106, 2011.

7. Pećina-Slaus N, Kafka A, Tomas D, Marković L, Okštajner PK, Sukser V and Krušlin B: Wnt signaling transcription factors TCF-1 and LEF-1 are upregulated in malignant astrocytic brain tumors. Histol Histopathol 29: 1557-1564, 2014.

8. MacDonald BT, Tamai K and He X: Wnt/beta-catenin signaling: Components, mechanisms and diseases. Dev Cell 17: 9-26, 2009.

9. Polakis P: Wnt signaling in cancer. Genes Dev 14: 1837-1851, 2000.

10. Fagotto F: Looking beyond the Wnt pathway for the deep nature of $\beta$-catenin. EMBO Rep 14: 422-433, 2013.

11. Kafka A, Bašić-Kinda S and Pećina-Slaus N: The cellular story of dishevelleds. Croat Med J 55: 459-467, 2014.

12. Hoang B, Moos M Jr, Vukicevic S and Luyten FP: Primary structure and tissue distribution of FRZB, a novel protein related to Drosophila frizzled, suggest a role in skeletal morphogenesis. J Biol Chem 271: 26131-26137, 1997.

13. Shi Y, He B, You L and Jablons DM: Roles of secreted frizzled-related proteins in cancer. Acta Pharmacol Sin 28 1499-1504, 2007

14. Bovolenta P, Esteve P, Ruiz JM, Cisneros E and Lopez-Rios J: Beyond Wnt inhibition: New functions of secreted Frizzled-related proteins in development and disease. J Cell Sci 121: 737-746, 2008 .

15. Jones SE and Jomary C: Secreted Frizzled-related proteins: Searching for relationships and patterns. Bioessays 24: 811-820, 2002.

16. Kawano Y and Kypta R: Secreted antagonists of the Wnt signalling pathway. J Cell Sci 116: 2627-2634, 2003.

17. Hirata H, Hinoda Y, Ueno K, Majid S, Saini S and Dahiya R Role of secreted frizzled-related protein 3 in human renal cell carcinoma. Cancer Res 70: 1896-1905, 2010.

18. Surana R, Sikka S, Cai W, Shin EM, Warrier SR, Tan HJ, Arfuso F, Fox SA, Dharmarajan AM and Kumar AP: Secreted frizzled related proteins: Implications in cancer. Biochim Biophys Acta 1845: 53-65, 2014

19. Mii Y and Taira M: Secreted Wnt 'inhibitors' are not just inhibitors: Regulation of extracellular Wnt by secreted Frizzled-related proteins. Dev Growth Differ 53: 911-923, 2011

20. Roth W, Wild-Bode C, Platten M, Grimmel C, Melkonyan HS, Dichgans J and Weller M: Secreted Frizzled-related proteins inhibit motility and promote growth of human malignant glioma cells. Oncogene 19: 4210-4220, 2000.

21. Harrison-Uy SJ and Pleasure SJ: Wnt signaling and forebrain development. Cold Spring Harb Perspect Biol 4: a008094, 2012.
22. Zhao X, Huang H, Chen Y, Liu Y, Zhang Z, Ma Q and Qiu M Dynamic expression of secreted Frizzled-related protein 3 (sFRP3) in the developing mouse spinal cord and dorsal root ganglia. Neuroscience 248: 594-601, 2013.

23. Sun J, Bonaguidi MA, Jun H, Guo JU, Sun GJ, Will B, Yang Z, Jang MH, Song H, Ming GL and Christian KM: A septo-remporal molecular gradient of sfrp3 in the dentate gyrus differentially regulates quiescent adult hippocampal neural stem activation. Mol Brain 8: 52, 2015.

24. Pazanin L: Histopatholoy of glial tumors. Medicina fluminensis 47: 157-166, 2011 (In Croatian).

25. Lee JL, Chang CJ, Wu SY, Sargan DR and Lin CT: Secreted frizzled-related protein 2 (SFRP2) is highly expressed in canine mammary gland tumors but not in normal mammary glands. Breast Cancer Res Treat 84: 139-149, 2004.

26. Abu-Jawdeh G, Comella N, Tomita Y, Brown LF, Tognazzi K, Sokol SY and Kocher O: Differential expression of frpHE: A novel human stromal protein of the secreted frizzled gene family, during the endometrial cycle and malignancy. Lab Investig 79: 439-447, 1999.

27. Drake J, Shearwood AM, White J, Friis R, Zeps N, Charles A and Dharmarajan A: Expression of secreted frizzled-related protein 4 (SFRP4) in primary serous ovarian tumours. Eur J Gynaecol Oncol 30: 133-141, 2009

28. Huang D, Yu B, Deng Y, Sheng W, Peng Z, Qin W and Du X: SFRP4 was overexpressed in colorectal carcinoma. J Cancer Res Clin Oncol 136: 395-401, 2010.

29. Paw I, Carpenter RC, Watabe K, Debinski W and Lo HW: Mechanisms regulating glioma invasion. Cancer Lett 362: 1-7, 2015.

30. Wang H, Xu T, Jiang Y, Xu H, Yan Y, Fu D and Chen J: The challenges and the promise of molecular targeted therapy in malignant gliomas. Neoplasia 17: 239-255, 2015.

31. Uren A, Reichsman F, Anest V, Taylor WG, Muraiso K, Bottaro DP, Cumberledge S and Rubin JS: Secreted frizzled-related protein-1 binds directly to Wingless and is a biphasic modulator of Wnt signaling. J Biol Chem 275: 4374-4382, 2000.

32. Xaviera CP, Melikovaa M, Chumana Y, Üren A, Baljinnyama B and Rubina JS: Secreted Frizzled-related protein potentiation versus inhibition of Wnt3a/ $\beta$-catenin signaling. Cell Signal 26: 94-101, 2014.

33. Lin K, Wang S, Julius MA, Kitajewski J, Moos M Jr and Luyten FP: The cysteine-rich frizzled domain of Frzb-1 is required and sufficient for modulation of Wnt signaling. Proc Natl Acad Sci USA 94: 11196-11200, 1997.

34. Bafico A, Gazit A,Pramila T, Finch PW, Yaniv A and Aaronson SA: Interaction of frizzled related protein (FRP) with Wnt ligands and the frizzled receptor suggests alternative mechanisms for FRP inhibition of Wnt signaling. J Biol Chem 274: 16180-16187, 1999.

35. Nikuševa Martić T, Pećina-Šlaus N, Kušec V, Kokotović T, Mušinović H, Tomas D and Zeljko M: Changes of AXIN-1 and beta-catenin in neuroepithelial brain tumors. Pathol Oncol Res 16: 75-79, 2010.

36. Zhang N, Wei P, Gong A, Chiu WT, Lee HT, Colman H, Huang H, Xue J, Liu M, Wang Y, et al: FoxM1 promotes $\beta$-catenin nuclear localization and controls Wnt target-gene expression and glioma tumorigenesis. Cancer Cell 20: 427-442, 2011.

37. Kahlert UD, Maciaczyk D, Doostkam S, Orr BA, Simons B, Bogiel T, Reithmeier T, Prinz M, Schubert J, Niedermann G, et al: Activation of canonical WNT/ $\beta$-catenin signaling enhances in vitro motility of glioblastoma cells by activation of ZEB1 and other activators of epithelial-to-mesenchymal transition. Cancer Lett 325: 42-53, 2012.

38. Cheng YY, Yu J, Wong JP, Man EP, To KF, Jin VX, Li J, Tao Q, Sung JJ, Chan JK and Leung WK: Frequent epigenetic inactivation of secreted frizzled-related protein 2 (SFRP2) by promoter methylation in human gastric cancer. Br J Cancer 97: 895-901, 2007.

39. Schiefer L, Visweswaran M, Perumal V, Arfuso F, Groth D, Newsholme P, Warrier S and Dharmarajan A: Epigenetic regulation of the secreted frizzled-related protein family in human glioblastoma multiforme. Cancer Gene Ther 21: 297-303, 2014.

40. Willert K, Brown JD, Danenberg E, Duncan AW, Weissman IL, Reya T, Yates JR III and Nusse R: Wnt proteins are lipid-modified and can act as stem cell growth factors. Nature 423: 448-452, 2003

41. Reya $\mathrm{T}$ and Clevers $\mathrm{H}$ : Wnt signaling in stem cells and cancer. Nature 434: 834-850, 2005. 